\title{
Examination of MCP-1 (CCL2) partitioning and presentation during transendothelial leukocyte migration
}

\author{
Lynne A Hardy ${ }^{1}$, Trevor A Booth ${ }^{1}$, Elaine $\mathrm{K} \mathrm{Lau}{ }^{2}$, Tracy $\mathrm{M} \mathrm{Handel}^{2}$, Simi $\mathrm{Ali}^{1}$ and \\ John A Kirby ${ }^{1}$ \\ ${ }^{1}$ Applied Immunobiology Group, School of Surgery and Reproductive Sciences, The Medical School, \\ University of Newcastle upon Tyne, UK and ${ }^{2}$ Department of Molecular and Cell Biology, University of \\ California, Berkeley, CA USA
}

\begin{abstract}
It is proposed that a chemokine concentration gradient promotes vectorial leukocyte migration across the vascular endothelium during inflammation. In this study, monocyte migration across a model endothelial monolayer was assessed at defined time-points after the addition of MCP-1 (CCL2). At each time-point transendothelial migration was quantified, medium from the apical and basal surface was collected for ELISA and monolayers were stained to detect both heparan sulfate and MCP-1. Statistically significant monocyte migration was observed within $60 \mathrm{~min}$ of chemokine addition to the basal surface of the endothelium and an asymmetric distribution of MCP-1 across the monolayer was observed at all time-points. Dual color immunofluorescence analysis demonstrated that MCP-1 was focused into heparan sulfate-containing domains on the apical surface of some of the endothelial cells. Furthermore, no uniform concentration gradient of chemokine was observed within the space between adjacent endothelial cells with apical MCP-1 application resulting in a staining pattern identical to that observed after basal application. The addition of a functional, monomeric form of MCP-1 produced a staining pattern identical to that observed using the wild-type protein, suggesting that localized chemokine oligomerization is not responsible for generating the focal chemokine distribution. Together, these data suggest that apical presentation of concentrated, chemokine-containing domains provides sufficient stimulus to promote transendothelial leukocyte migration in the absence of the formation of a formal haptotactic concentration gradient between endothelial cells.
\end{abstract}

Laboratory Investigation (2004) 84, 81-90, advance online publication, 1 December 2003; doi:10.1038/labinvest.3700007

Keywords: chemotaxis; glycosaminoglycans; confocal microscopy; immunofluorescence; MCP-1; heparan sulfate

Chemokines are small, predominantly proinflammatory proteins with roles in leukocyte trafficking and homeostasis as well as transplant rejection and inflammatory disease. There are two major (CXC and $\mathrm{CC})$ and two minor $\left(\mathrm{CX}_{3} \mathrm{C}\right.$ and $\left.\mathrm{C}\right)$ families, classified by the location of the first two cysteine residues within the primary protein sequence. ${ }^{1,2}$

Although there is a large degree of sequence diversity between members of the chemokine superfamily, there is a conserved three-dimensional structure consisting of a short mobile amino term-

Correspondence: Professor JA Kirby, Applied Immunobiology Group, School of Surgical and Reproductive Sciences, The Medical School, University of Newcastle upon Tyne, Newcastle upon Tyne, UK.

E-mail: J.A.Kirby@ncl.ac.uk

Received 21 August 2003; revised 19 September 2003; accepted 09 October 2003; published online 1 December 2003 inal, a stable core region composed of three antiparallel $\beta$-sheets and a C-terminal $\alpha$-helix. Interaction with specific chemokine receptors (all members of the seven trans-membrane spanning Gprotein coupled receptor family) is via the $\mathrm{N}$ terminal and an exposed loop of the core between the second and third conserved cysteines, with the $\mathrm{N}$-terminus critical for signal transduction. ${ }^{2}$ Interaction with a low-affinity receptor, the cell surface glycosaminoglycans (GAG), is usually mediated via the C-terminus of the protein and often via a specific BBXB motif. ${ }^{3,4}$

Chemokines display a range of affinities for the various glycosaminoglycan components of cell surface and extracellular proteoglycans, predominantly binding to the highly sulfated heparin-like species, heparan sulfate. ${ }^{5}$ The ability to discriminate between different GAG molecules is believed to reside in paired glutamic acid residues within putative 
GAG-binding sites. ${ }^{6}$ Heparan sulfate itself is a highly heterogeneous molecule. ${ }^{7}$ Indeed, the subtle variations in GAG structure may help to determine the specific localization of chemokines within certain tissues as every cell type may have a distinct pattern of GAG expression. ${ }^{8}$

MCP-1 (CCL2), a potent monocyte attractant, is a member of the CC subfamily. ${ }^{9}$ It was originally isolated from mouse $3 \mathrm{~T} 3$ fibroblasts ${ }^{10}$ and its expression can be induced by a variety of growth factors, cytokines and bacterial lipopolysaccharide. Truncation of the N-terminal of the protein leads to a loss of function, ${ }^{11-14}$ with residues $13-35$ also involved in the recognition of its receptor, CCR2. ${ }^{15}$ Monocyte chemotaxis in response to receptor ligation is pertussis toxin sensitive, suggesting that the receptor is coupled to $\mathrm{G}_{\alpha \mathrm{i}}{ }^{16}$ The MAP kinases ERK1 and ERK2, the stress-activated kinases JNK1 and p38, phospholipase C, two isoforms of PI3-kinase (p85/p110 and C2 $\alpha$ ) and the Janus kinase JAK2, have all been implicated in MCP-1 signal transduction. ${ }^{17-20}$ MCP-1 is known to trigger the firm arrest of rolling monocytes on endothelial monolayers expressing Eselectin $^{21}$ and may have a role in spreading and shape change of monocytes attached to the endothelium. ${ }^{22}$ Lys $^{58}$ and $\mathrm{His}^{66}$ are known to be critical to the interaction between MCP-1 and heparan sulfate, ${ }^{23}$ and competition with heparin attenuates its chemotactic potential. ${ }^{24}$

Transendothelial migration of leukocytes in response to chemokines is a multistep process mediated by a variety of proteins. ${ }^{25,26}$ Initial rolling of the leukocyte along the endothelial surface, mediated by cell surface selectins, is upregulated to firm adhesion by the leukocyte integrin family and their receptors on the endothelial surface after exposure to GAG-immobilized chemokine. Leukocytes then migrate through the endothelium in a process known as diapedesis, by a coordinated rearrangement of cell-cell contacts. ${ }^{27,28}$

It has been proposed that a cell surface, or haptotactic, gradient of chemokine is responsible for the directed migration of leukocytes from the vascular lumen into the tissue compartment. Indeed, in static in vitro experiments, a soluble gradient does elicit the vectorial migration of a variety of purified leukocytes from areas of low to high protein concentration. ${ }^{20,24,29}$ However, recent work by Cinamon et $a l^{30}$ and others ${ }^{31,32}$ has highlighted the importance of both shear flow and apical presentation of chemokine in the direction of diapedesis in model systems.

In order to elucidate the presence of the proposed chemotactic gradient, an in vitro model system was developed based on the standard trans-well assay followed by immunofluorescent staining techniques to localize the prototypical CC chemokine MCP-1 during leukocyte migration. The role of apical presentation of chemokine within the model system was also investigated, as was the role of multimerization in the formation of focal chemokine domains on the apical surface of the endothelium.

\section{Materials and methods}

\section{Cell Lines and Monolayer Culture}

The EA.hy926 hybridoma cell line ${ }^{33}$ was a gift from Dr Cora-Jean Edgell (University of North Carolina, USA) and was used to provide a reproducible model of vascular endothelium. Cells (125 000) were added to a trans-well chamber containing a $3 \mu \mathrm{m}$ membrane (Falcon, BD Biosciences) and cultured to confluence in DMEM containing $10 \%$ fetal calf serum, $100 \mathrm{U}$ penicillin and $0.1 \mathrm{mg} / \mathrm{ml}$ streptomycin $\left(\mathrm{DMEM}^{+}\right)$ (Sigma, UK) at $37^{\circ} \mathrm{C}$ in a $5 \% \mathrm{CO}_{2}$ atmosphere. The cells were then washed in $\mathrm{DMEM}^{+}$prior to transfer to the assay wells.

\section{Preparation of Peripheral Blood Mononuclear Cells (PBMC)}

Anticoagulated fresh whole blood $(20-30 \mathrm{ml})$ was extracted from healthy donors and separated over a Ficoll Paque-Plus gradient (Amersham Biosciences, UK) as previously described. ${ }^{24}$.The cells recovered from the interface were washed twice with DMEM prior to being resuspended at a concentration of $2 \times 10^{6}$ cells $/ \mathrm{ml}$.

\section{Standard Trans-well Chemotaxis Assay}

Trans-wells containing a confluent monolayer of resting endothelial cells were transferred to 24-well plates containing MCP-1 (Peprotech, UK) at a concentration of $12.5 \mathrm{nM}$. in $\mathrm{DMEM}^{+}$in the lower reservoir. Freshly isolated PBMC $\left(1 \times 10^{6}\right)$ were added to the upper chamber of the trans-well and the assembly was incubated at $37^{\circ} \mathrm{C}$ for 30,60 and $90 \mathrm{~min}$. As a negative control for background migration, $\mathrm{DMEM}^{+}$was substituted for chemokine in the lower chamber. Cells that had migrated from the upper chamber to the lower chamber were then collected for staining and analyzed by flow cytometry.

\section{Apical Application of Chemokine}

MCP-1 at a concentration of $12.5 \mathrm{nM}$. in serum-free DMEM was applied to an EA.hy926 monolayer for $1 \mathrm{~h}$ at $37^{\circ} \mathrm{C} .{ }^{34}$ Monolayers were then washed twice for 5 min in serum free DMEM at room temperature prior to transfer to the standard migration assay system. Migration was for $90 \mathrm{~min}$ at $37^{\circ} \mathrm{C}$ to allow direct comparison to the standard assay described above.

\section{P8A Chemotaxis Assay}

The monomeric MCP-1 mutant $\mathrm{P} 8 \mathrm{~A}^{35}$ was substituted for wild-type MCP-1 in the standard chemotaxis 
assay at a concentration of $12.5 \mathrm{nM}$. Migration was allowed to continue for $90 \mathrm{~min}$ at $37^{\circ} \mathrm{C}$ with the relevant negative and positive controls.

\section{Flow Cytometry}

Migrating cells were stained for $30 \mathrm{~min}$ at $4{ }^{\circ} \mathrm{C}$ with a dual-labelled anti-CD45/anti-CD14 antibody to detect the monocytic population (DakoCytomation). The samples were then analyzed on a FacScan machine using LYSIS II software (BD Biosciences). An equal quantity of fluorescent beads was added to each sample to allow direct ratiometric comparison between tubes (Flow-count fluorospheres, Beckman Coulter, USA). The data collected were further analyzed using FCS Express (DeNovo Software, Ontario, Canada) and the chemotactic index calculated as (monocytic migratory cells)/(monocytic migratory cells + fluorescent spheres) or R2/ (R2 + R3) (Figure 1b).

\section{MCP-1 ELISA}

Supernatants from both the upper and lower chambers were retained after chemotaxis to
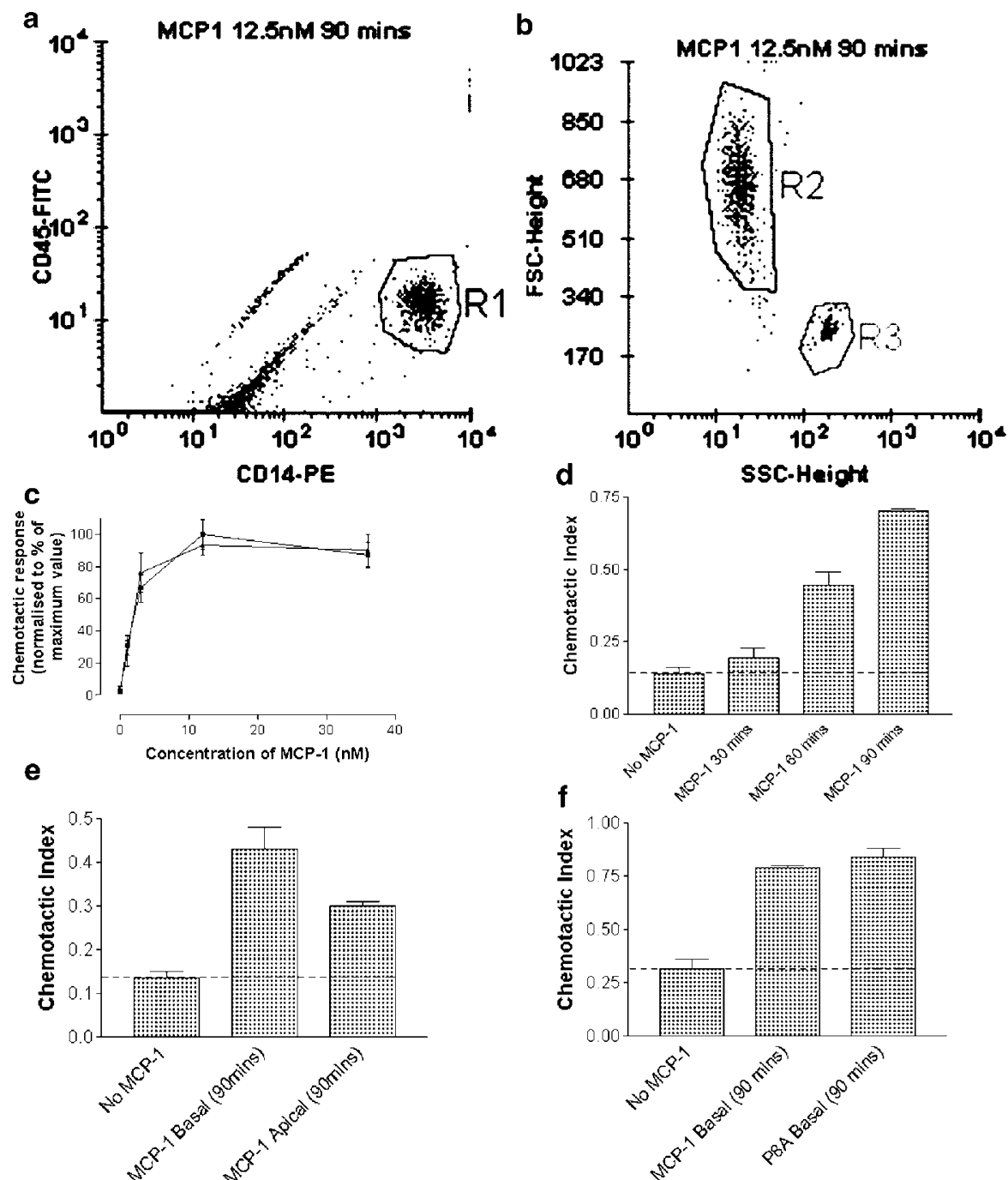

Figure 1 Transendothelial migration across an intact endothelial monolayer. (a) Identification of the monocytic cell population by CD45/ CD14 staining. R1 represents the dual-stained population of interest. (b) Quantification of migrating monocytes in response to $12.5 \mathrm{nM}$ MCP-1 for $90 \mathrm{~min}$; R2 represents the migrating monocytes and R3 the control population of fluorescent beads. (c) Determination of the optimum concentration of MCP-1 for transendothelial migration. The figure shows representative data from two separate experiments with three replicates for each condition; the points represent mean values and the error bars show the standard error. (d) Chemotaxis in response to $12.5 \mathrm{nM}$ MCP-1 in the basal chamber. The figure shows representative data from one of three separate experiments with three replicates for each condition; the points represent the mean values and the error bars show the standard error. (e) Chemotaxis assay comparing the results produced following the addition of $12.5 \mathrm{nM} \mathrm{MCP-1}$ to either the basal or apical chambers. The figure shows representative data from one of four separate experiments with three replicates for each condition; the points represent the mean values and the error bars show the standard error. (f) Chemotaxis assay comparing the results produced following the addition of either $12.5 \mathrm{nM}$ P8A or $12.5 \mathrm{nM}$ wild-type MCP-1. The figure shows representative data from one of six separate experiments with three replicates for each condition; the points represent the mean values for this experiment; the error bars indicate the standard error. 
determine the localization of MCP-1 in free solution above and below the monolayer. ELISA was performed according to the manufacturer's protocol using the Duoset ELISA development kit (R\&D Systems). The plates were assayed on an OpsysMR 96-well plate reader (Dynex Technologies, Ashford, Middlesex, UK) at $450 \mathrm{~nm}$ and concentrations determined from a standard curve using the Prism software package.

\section{Immunofluorescent Staining of Monolayers}

Chemokine-treated monolayers were fixed in $2 \%$ paraformaldehyde at $4^{\circ} \mathrm{C}$ for $1 \mathrm{~h}$ to prevent protein migration during subsequent stages. After washing in phosphate-buffered saline (PBS), the cells were blocked in $10 \%$ horse serum/PBS (Sigma, UK) for $15 \mathrm{~min}$ at room temperature. The monolayers were washed with PBS between each subsequent step and all incubations were at room temperature.

Monoclonal anti-heparan sulfate antibodies 10E4 and HepSS-1 (Seikagaku, USA) were used at a concentration of $1 \mu \mathrm{g} / \mathrm{ml}$ and goat polyclonal antiMCP-1 (Santa Cruz) was used at a concentration of $2 \mu \mathrm{g} / \mathrm{ml}$. The primary antibodies were applied together in $10 \%$ horse serum/PBS for $1 \mathrm{~h}$. The monolayers were then blocked with $3 \%$ rabbit serum/PBS (Sigma, UK) for $15 \mathrm{~min}$ followed by a 1/100 dilution of rabbit-anti-goat-TRITC (RAGTRITC; Sigma, UK) in $3 \%$ rabbit serum/PBS for $1 \mathrm{~h}$. Cells were then blocked again in $3 \%$ goat serum/ PBS (GAM-FITC; Sigma, UK) for $15 \mathrm{~min}$ prior to the final secondary antibody application of goat-antimouse-FITC in $3 \%$ goat serum/PBS (1/100 dilution; BD Biosciences). Monolayers were cut from the trans-well chambers and mounted onto slides in fluorescent mounting medium (DakoCytomation) prior to confocal microscopy. Negative controls for nonspecific binding by the secondary antibodies were performed as described above, using $10 \%$ horse serum/PBS substituted for the primary antibody.

\section{Confocal Microscopy}

All slides were analyzed on a Leica TCS SP2 UV using v2.00. software (build 0871) under a $\times 63$ oil immersion objective. An argon ion laser (excitation $488 \mathrm{~nm}$, emission collection window $503-563 \mathrm{~nm}$ ) was used for FITC detection and a $\mathrm{He} / \mathrm{Ne}$ laser (excitation $543 \mathrm{~nm}$, emission collection window 552-617 nm) was used for TRITC detection. Fluorescence levels were set for each experiment using a positive control slide, usually detection of MCP-1 $60 \mathrm{~min}$ after basal application, and all other slides in that series were analyzed using the same settings to allow direct comparison within each experiment. Images were captured sequentially frame by frame to eliminate crosstalk, using a 488/543 dual dichroic filter and a pinhole setting of 1.2AE. Images captured on the confocal microscope were then composited using Adobe Photoshop to give an overlapping image for both MCP-1 and heparan sulfate staining.

\section{Radioligand Assay of MCP-1 Binding to Cultured Endothelial Cells}

A series of radioligand binding assays were performed to assess the effects of heparin or chondroitin sulfate-C (Sigma) on MCP-1 binding to endothelial cells in culture. In these assays, EA.hy926 cells were cultured to confluency in 96well plates and ${ }^{125} \mathrm{I}$-MCP-1 was added at a concentration of $100 \mathrm{pM}$ in the presence of either heparin or chondroitin sulfate-C at concentrations between 0 and $500 \mu \mathrm{g} / \mathrm{ml}$. After incubation at $37^{\circ} \mathrm{C}$ for $90 \mathrm{~min}$, the plate was washed with HBSS containing $10 \mathrm{mM}$ HEPES and $0.1 \%$ BSA (Sigma) to remove any unbound ligand. The cells were then lysed by incubation at $37^{\circ} \mathrm{C}$ for $2 \mathrm{~h}$ in $1 \%$ SDS/1 M $\mathrm{NaOH}$ and transferred to test tubes; the radioactivity was then measured (Clinigamma; Wallac, Milton Keynes, Beds, UK). The amount of protein extracted from each well was assayed (BCA protein assay; Pierce Chemical Co., Perbio Science Ltd, Cheshire, UK) and the data were normalized to \% maximal cpm per mg protein.

\section{Results}

Time-course of Monocyte Chemotaxis in Response to MCP-1

EA.hy926 cells were chosen to represent intact endothelium in a reproducible in vitro model system of transendothelial migration. These cells were formed by the fusion of human umbilical vein endothelial cells (HUVECs) with the lung carcinoma cell line A549 (ATCC CCL 185), and express many features specific to endothelial cells including Weibel-Palade bodies, endothelial cell surface markers and the capacity for adhesion to immune cells and for antigen presentation to $\mathrm{T}$ cells. ${ }^{33,36,37}$ They also express large amounts of both $\mathrm{N}$ - and $\mathrm{O}$-sulfated heparan sulfate on their cell surface as detected by immunofluorescence flow cytometry (data not shown) and have been used previously by this group and others to model endothelial/cytokine interactions. ${ }^{38-40}$ Importantly, immunofluorescence microscopy demonstrated that they lack cell surface expression of CCR2, the cognate receptor for MCP-1 (data not shown).

Monocytes were identified within the PBMC population by dual staining for the general leukocyte marker CD45 and the monocyte-specific marker CD14 (Figure 1a); monocytes were counted by reference to the signal generated by a known concentration of beads (Figure 1b). The optimum concentration of MCP-1 in the subendothelial 
compartment required for chemotaxis was found to be $12.5 \mathrm{nM}$ (Figure 1c); this concentration was used in all subsequent experiments. The migration of monocytes across an EA.hy926 monolayer was found to be time dependent, with migration detectable in almost all cases after $60 \mathrm{~min}$ (dependent on donor) and reproducible by $90 \mathrm{~min}$ (Figure 1d). Apical application of MCP-1 to EA.hy926 monolayers for 90 min prior to the addition of PBMC also elicited reproducible migration of monocytes (Figure 1e), but to a lesser extent than that seen in the standard assay with basal chemokine. Chemotaxis assays were also performed in the presence of the monomeric MCP-1 mutant P8A (35) to assess its ability to sustain transendothelial migration and to determine if oligomerisation was responsible for the focal staining pattern observed by confocal microscopy (see below). The transendothelial monocyte chemotaxis observed in response to P8A was similar to that produced by the wild-type protein (Figure 1f).

\section{Asymmetric Distribution of Soluble MCP-1 across a Monolayer}

Redistribution of MCP-1 across an intact EA.hy926 monolayer was rapid and asymmetric, with stable concentrations in both compartments being formed within $60 \mathrm{~min}$ of the addition of chemokine to the subendothelial chamber (Figure 2a, b). The level of MCP-1 above naked membranes (no cell monolayer; Figure 2a) was much higher than above an endothelial monolayer-covered filter, indicating that the cells provide a barrier to free diffusion of chemokine into the upper chamber. The EA.hy926 cells produce low levels of MCP-1 endogenously, but the chemokine generated in this way is distributed equally on both sides of the monolayer (Figure 2a, b), and does not induce significant chemotaxis. ${ }^{41,42}$ When the localization of MCP-1 was analyzed by ELISA after apical application of chemokine, levels of MCP-1 free in solution above the monolayer were similar to those seen in the negative control, suggesting that any cell migration observed in this system was driven solely by surface-bound MCP-1.

Although the P8A mutant protein could be detected by ELISA, values were lower than for an equivalent amount of wild-type protein, suggesting that the detection antibody epitope had been marginally affected by the mutation. However, as with the wild-type protein, EA.hy926 cells provided an effective barrier to free diffusion into the upper chamber of P8A, the obligate monomeric MCP-1 mutant (Figure 2c).

\section{Distribution of MCP-1 on EA.hy926 Cells by Immunofluorescence Staining}

Following subendothelial exposure to MCP-1, immunofluorescence of nonpermeabilized cells demonstrated that heparan sulfate was relatively
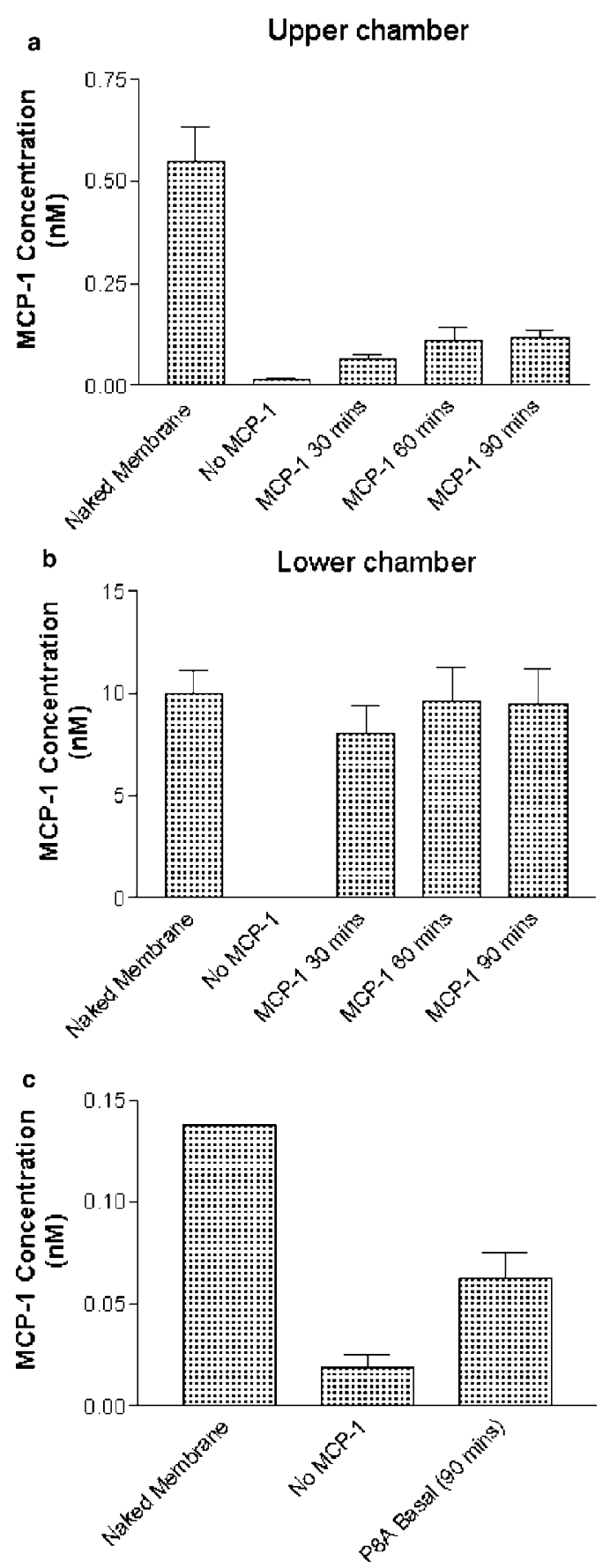

Figure 2 Asymmetric localization of MCP-1 following the addition of $12.5 \mathrm{nM}$ MCP-1 to the subendothelial compartment. (a) Concentrations of MCP-1 in the upper experimental chamber. (b) Concentrations of MCP-1 in the lower experimental chamber. Mean data in (a) and (b) were derived from six separate experiments with three replicates for each condition; the error bars show the standard error. (c) P8A concentrations in the upper chamber of the trans-well system after basal application of the mutant protein for $90 \mathrm{~min}$ at $37^{\circ} \mathrm{C}$. The representative data shown are from one of five separate experiments with three replicates for each condition; mean values are shown together with the standard error.

uniformly expressed across the surface of the endothelial cells (Figure 3a; FITC stain). By contrast, the surface of cells in the same field showed a 
punctate distribution of MCP-1, with some cells expressing high concentrations of this protein and others expressing little or none (Figure 3a; TRITC stain). Endothelial monolayers that had not been exposed to exogenous chemokine showed no significant expression of cell surface MCP-1 (data not shown); this is consistent with a previous study that demonstrated little MCP-1 production by resting EA.hy926 cells. $^{24}$ The antibodies used for the detection of heparan sulfate are specific for epitopes known to be important in MCP-1 binding: 10E4 is specific for molecules containing N-sulfated glucosamine, ${ }^{43}$ while HepSS-1 binds to O-sulfated, $\mathrm{N}$-acetylated glucosamine residues linked to glucuronic acid. ${ }^{44}$

Reconstructed two-color $X-Z$ sections allowed direct visualization of the distribution of both heparan sulfate and MCP-1 within endothelial monolayers after treatment for $60 \mathrm{~min}$ with basally applied chemokine (Figure 3b, c). Heparan sulfate was found on the apical and lateral surfaces of the endothelial cells, with little evidence of local concentration. By contrast, MCP-1 was restricted to only $22 \%$ (42 of 192 cells examined in 64 preparations) of the endothelial cells; the presence of this chemokine was often, but not exclusively, coincidental with the presence of a pore in the underlying filter support. Of these cells, $26 \%$ showed a generalized apical cell surface expression of the chemokine with some evidence of distribution between adjacent cells (Figure 3b), possibly suggesting a paracellular transport route. However, $74 \%$ of the chemokine-expressing cells showed evidence of the concentration of MCP-1 within a single site on the apical cell surface (Figure 3c); the yellow staining observed on the composite image is indicative of colocalization of heparan sulfate and MCP-1 at these sites.

Following apical application of MCP-1, stained monolayers exhibited the same punctate staining pattern for the chemokine on plan view as had been observed following basal application of the chemo- a

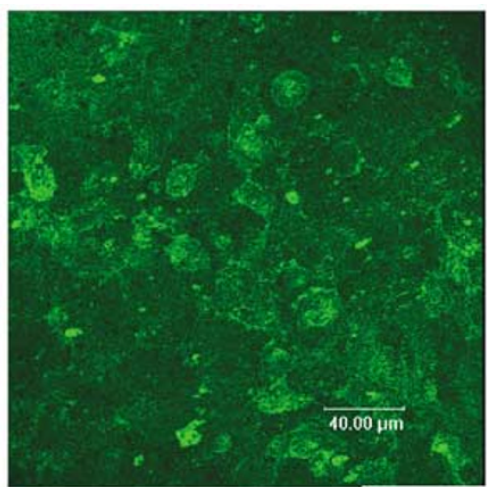

b
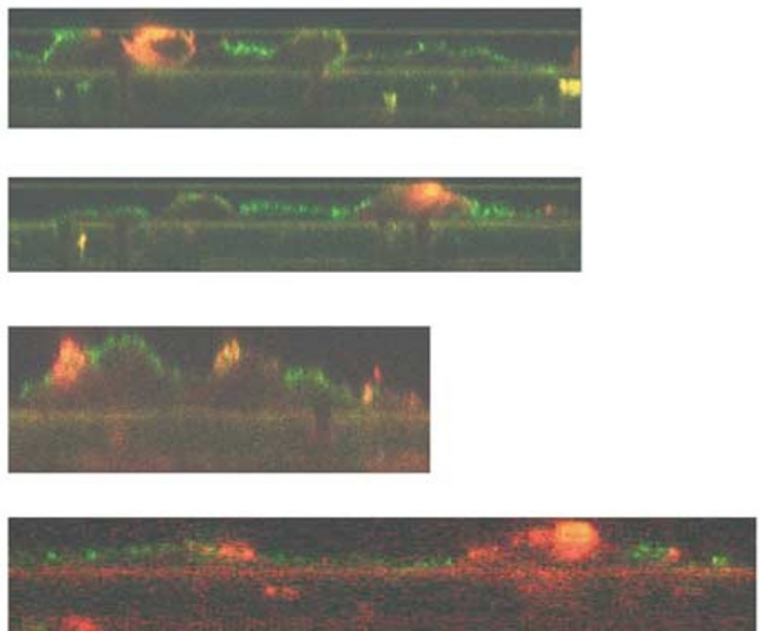

Figure 3 Immunofluorescence detection of MCP-1 and heparan sulfate by confocal microscopy; the green channel shows FITC-stained heparan sulfate and the red channel represents TRITC-stained MCP-1. (a) Representative paired $X-Y$ images showing relatively uniform distribution of heparan sulfate (green) but punctate distribution of MCP-1 (red) on the endothelial cell surface following incubation with MCP-1 in the subendothelial compartment. (b,c) Two-color X-Z sectional images showing characteristic distributions of MCP-1 associated with single cells (b) and concentrated into discrete regions of the apical cell surface (c) following incubation with subendothelial MCP-1; yellow regions indicate colocalization of heparan sulfate and MCP-1. (d) Immunofluorescence staining after apical application of $12.5 \mathrm{nM}$ MCP-1 for $60 \mathrm{~min}$ showing focal distribution of chemokine on the apical cell surface, which is similar to that produced by basal chemokine application. (e) Immunodetection of the P8A monomeric mutant MCP-1 following basal application at $12.5 \mathrm{nM}$; again, the staining shows a focal distribution on the apical cell surface. 
kine (data not shown). Examination of composite $X-$ $Z$ sections again demonstrated the presence of focal accumulations of MCP-1 on $43 \%$ (13 of 30 cells examined in 12 preparations) of cells (Figure $3 \mathrm{~d}$ ); this provides evidence that focal chemokine presentation is not a consequence of the mechanism of MCP-1 redistribution across an endothelial monolayer from the basal compartment.

A candidate mechanism for the development of focal concentrations of MCP-1 on the apical surface of endothelial cells is the formation of chemokine oligomers, ${ }^{42}$ a process that is enhanced by the presence of heparan-like GAG species. ${ }^{45}$ In order to investigate this possibility, an obligate monomeric MCP-1 mutant P8A was added to the basal chamber and immunoreactive protein was subsequently identified on the apical surface of the model endothelium. As for the wild-type chemokine, it was found that the mutant protein was largely concentrated into focal regions on the apical surface of $26 \%$ ( 23 of 87 cells examined in 29 preparations) of the cells (Figure 3e). This suggests that the formation of chemokine oligomers is not required for focal presentation of chemokines on the apical surface of the endothelial monolayer.

\section{Competition of Cell Surface MCP-1 Binding by Heparin}

In order to explore the interaction between MCP-1 and GAG on the apical surface of the model endothelium, a series of radioligand competition assays were performed in the presence of heparin and the structurally unrelated GAG, chondroitin sulfate-C (Figure 4). It was found that titration into the medium of an increasing concentration of soluble heparin, but not of chondroitin sulfate-C, inhibited binding of ${ }^{125} \mathrm{I}-\mathrm{MCP}-1$ to the surface of confluent cell cultures. This provides direct evidence that MCP-1 binds normally to heparin-like GAG on the cell surface. Importantly, the concentration at which soluble heparin reproducibly inhibited MCP-1 binding to the cell surface $(250 \mu \mathrm{g} / \mathrm{ml})$ was similar to that estimated for heparan sulfate within the proteoglycan layer on the endothelial cell surface $(200-500 \mu \mathrm{g} / \mathrm{ml}) .^{7,46}$

\section{Discussion}

Chemokine expression plays a crucial role during the recruitment of immune cells to areas of inflammation by promoting migration across the vascular endothelium. Vectorial migration in response to a chemokine concentration gradient has been thought to drive this recruitment, but blood flow would prevent the formation of a stable gradient within the vascular lumen. Consequently, the discovery that chemokines are bound by cell surface GAG gave rise to the theory that haptotactic, or cell-bound, concentration gradients provide vectorial information

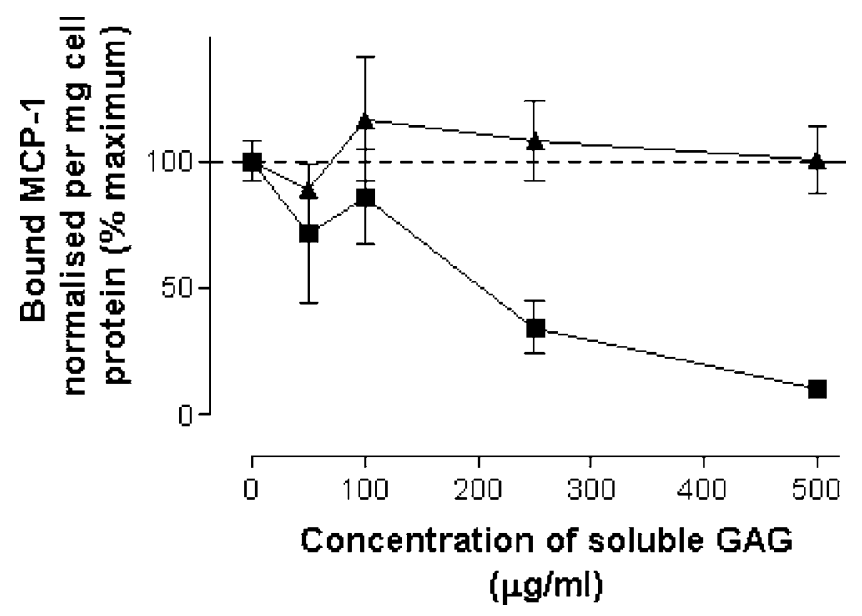

Figure 4 Competition of endothelial cell surface bound ${ }^{125}$ I-MCP1 by the soluble GAG species heparin (ם) and chondroitin sulfate-C $(\boldsymbol{\Lambda})$. The mean data are representative of three separate experiments with four replicates for each condition and the error bars show the standard error.

in vivo. ${ }^{5,47}$ A variety of experiments have shown that GAG are critically important for chemokine activity, ${ }^{3,5,23,48}$ with loss of cell surface heparan sulfate severely limiting chemokine activity. ${ }^{34,42,49}$

Soluble chemokine concentration gradients have been shown to direct immune cell migration in vitro. During inflammation in vivo, it is likely that chemokines produced by subendothelial tissues contribute to the recruitment of blood-borne leukocytes following redistribution and presentation on the apical surface of the endothelium. However, before the present study, neither this process nor the potential development of a transendothelial haptotactic gradient has been directly visualized after basal application of chemokine.

In this study, confocal analysis showed that MCP1 was predominantly sequestered on the apical cell surface within $60 \mathrm{~min}$ of application below a confluent model endothelium. Although the chemokine staining observed between some of the endothelial cells provided data consistent with a paracellular transport route, a classical transendothelial haptotactic concentration gradient was not identified. Indeed, the highest concentration of cell-associated chemokine appeared on the apical surface at those time-points for which significant monocyte migration was observed.

As the cells were not permeabilized to allow efficient detection of intracellular chemokine, it is not possible to exclude the contribution of a transcellular transport route, such as that observed during IL-8 redistribution. ${ }^{50}$ However, the focal distribution of MCP-1 observed on the apical cell surface is unlikely to be related to discharge of transport vesicles, as a similar pattern of focal chemokine distribution was observed following apical application of MCP-1.

The finding that the sequestration of MCP-1 onto the endothelial cell surface could be competitively 
inhibited by addition of soluble heparan-like GAG was consistent with the observed colocalization of MCP-1 with cell surface heparan sulfate. However, it is clear that the focal distribution of MCP-1 was not regulated solely by the presence of similarly distributed heparan sulfate as this GAG was found across the entire cell surface. A similar focal distribution has been reported for cell surface FGF$2 ;^{51}$ in this case, the growth factor complexes with heparan sulfate to form protein-rich 'microdomains', which are distinct from the lipid rafts involved in FGF-2 signal transduction.

The capacity of heparan sulfate to bind chemokines is known to depend on the distribution and chemical composition of sulfated polyanionic motifs within the GAG chain. Although the precise structure of MCP-1-binding motifs has not been defined, there is a clear requirement for both $\mathrm{N}$ - and O- sulfation. ${ }^{5}$ Recent work from our group has shown that the availability of chemokine-binding motifs can depend on changes in the expression of sulfotransferase enzymes, such as $\mathrm{N}$-deacetylase $/ \mathrm{N}$ sulfotransferase-1. ${ }^{52}$ Hence, chemical microheterogeneity within the cell surface heparan sulfate population provides a possible explanation for the focal accumulation of MCP-1.

An alternative explanation is that the cell surface distribution of the proteoglycan core proteins to which heparan sulfate chains are attached could focus the availability of suitable MCP-1-binding sites. For example, members of the glypican family are attached to the cell surface by GPI anchors and are known to be sequestered in lipid rafts, while members of the syndecan family are linked to the cytoskeleton $^{53}$ and are differentially sequestered on the cell surface. ${ }^{7,8}$

It has been proposed that cell surface GAG form a scaffold within which chemokines are assembled into multimers. ${ }^{45}$ While MCP-1 has been found as a dimer in solution at concentrations in the physiological range and as a tetramer at higher concentrations, this appears to have no functional significance as obligate monomers show a normal activity. ${ }^{35,42,44}$ Chemokine multimerization within the cell surface GAG layer could provide a further explanation for the focal distribution observed for MCP-1. However, this possibility can be discounted because a focal distribution of MCP-1 was also observed following treatment of the model endothelium with a monomeric MCP-1 mutant.

Following definition of the apical pattern of MCP1 distribution under standard assay conditions, the distribution and biological activity of apically applied MCP-1 were also assessed. As seen for RANTES (CCL5), apically bound chemokine was sufficient to promote leukocyte migration, although to a smaller extent to that observed under standard assay conditions. ${ }^{34}$ These observations are consistent with a recent study that has shown that, at least for lymphocytes, a transendothelial concentration gradient is not necessary for transendothelial migra- tion, with apically bound chemokine providing a sufficient cue. ${ }^{30}$

In this study, we demonstrate that MCP-1 is rapidly redistributed from the basal to the apical surface of a model endothelium leaving no evidence of a haptotactic concentration gradient between the cells constituting the monolayer. Significantly, the chemokine presented on the apical endothelial cell surface is focused into discrete regions. While this interaction is dependent on heparan sulfate, it is not regulated solely by the presence of this GAG. We suggest that this provides a mechanism for concentrating chemokines, which are produced in small quantities by subendothelial tissues, onto the apical surface of the endothelium in order to promote efficient activation and recruitment of blood-borne leukocytes.

\section{Acknowledgement}

This study was supported by the British Heart Foundation (PG/99141), the National Institutes of Health and the American Heart Association.

\section{References}

1 Rollins BJ. Chemokines. Blood 1997;90:909-928.

2 Baggiolini M. Chemokines and leukocyte traffic. Nature 1998;392:565-568.

3 Webb LM, Ehrengruber MU, Clark-Lewis I, et al. Binding to heparan sulfate or heparin enhances neutrophil responses to interleukin 8. Proc Natl Acad Sci USA 1993;90:7158-7162.

4 Lortat-Jacob H, Grosdidier A, Imberty A. Structural diversity of heparan sulfate binding domains in chemokines. Proc Natl Acad Sci USA 2002;99: 1229-1234.

5 Kuschert GS, Coulin F, Power CA, et al. Glycosaminoglycans interact selectively with chemokines and modulate receptor binding and cellular responses. Biochemistry 1999;38:12959-12968.

6 Witt DP, Lander AD. Differential binding of chemokines to glycosaminoglycan subpopulations. Curr Biol 1994;4:394-400.

7 Bernfield M, Gotte M, Park PW, et al. Functions of cell surface heparan sulfate proteoglycans. Annu Rev Biochem 1999;68:729-777.

8 Park PW, Reizes O, Bernfield M, et al. Cell surface heparan sulfate proteoglycans: selective regulators of ligand-receptor encounters. J Biol Chem 2000;275: 29923-29926.

9 Gu L, Tseng SC, Rollins BJ. Monocyte chemoattractant protein-1. Chem Immunol 1999;72:7-29.

10 Cochran BH, Reffel AC, Stiles CD. Molecular cloning of gene sequences regulated by platelet-derived growth factor. Cell 1983;33:939-947.

11 Zhang YJ, Rutledge BJ, Rollins BJ. Structure/activity analysis of human monocyte chemoattractant protein1 (MCP-1) by mutagenesis. Identification of a mutated protein that inhibits MCP-1-mediated monocyte chemotaxis. J Biol Chem 1994;269:15918-15924. 
12 Gong JH, Clark-Lewis I. Antagonists of monocyte chemoattractant protein-1 identified by modification of functionally critical $\mathrm{NH}_{2}$-terminal residues. J Exp Med 1995;181:631-640.

13 Steitz SA, Hasegawa K, Chiang SL, et al. Mapping of MCP-1 functional domains by peptide analysis and site-directed mutagenesis. FEBS Lett 1998;430: 158-164.

14 Jarnagin K, Grunberger D, Mulkins M, et al. Identification of surface residues of the monocyte chemotactic protein 1 that affect signaling through the receptor CCR2. Biochemistry 1999;38:16167-16177.

15 Hemmerich S, Paavola C, Bloom A, et al. Identification of residues in the monocyte chemotactic protein-1 that contact the MCP-1 receptor, CCR2. Biochemistry 1999;38:13013-13025.

16 Sozzani S, Luini W, Molino M, et al. The signal transduction pathway involved in the migration induced by a monocyte chemotactic cytokine. J Immunol 1991;147:2215-2221.

17 Mellado M, Rodriguez-Frade JM, Aragay A, et al. The chemokine monocyte chemotactic protein 1 triggers Janus kinase 2 activation and tyrosine phosphorylation of the CCR2B receptor. J Immunol 1998;161:805-813.

18 Turner SJ, Domin J, Waterfield MD, et al. The CC chemokine monocyte chemotactic peptide-1 activates both the class I p85/p110 phosphatidylinositol 3kinase and the class II PI3K-C2alpha. J Biol Chem 1998;273:25987-25995.

19 Cambien B, Pomeranz M, Millet MA, et al. Signal transduction involved in MCP-1-mediated monocytic transendothelial migration. Blood 2001;97:359-366.

20 Wain JH, Kirby JA, Ali S. Leucocyte chemotaxis: examination of mitogen-activated protein kinase and phosphoinositide 3-kinase activation by monocyte chemoattractant proteins-1, $-2,-3$ and -4 . Clin Exp Immunol 2002;127:436-444.

21 Gerszten RE, Garcia-Zepeda EA, Lim YC, et al. MCP-1 and IL 8 trigger firm adhesion of monocytes to vascular endothelium under flow conditions. Nature 1999;398:718-723.

22 Weber KS, von Hundelshausen P, Clark-Lewis I, et al. Differential immobilization and hierarchical involvement of chemokines in monocyte arrest and transmigration on inflamed endothelium in shear flow. Eur J Immunol 1999;29:700-712.

23 Chakravarty L, Rogers L, Quach T, et al. Lysine 58 and histidine 66 at the C-terminal alpha-helix of monocyte chemoattractant protein-1 are essential for glycosaminoglycan binding. J Biol Chem 1998;273:29641-29647.

24 Douglas MS, Ali S, Rix DA, et al. Endothelial production of MCP-1: modulation by heparin and consequences for mononuclear cell activation. Immunology 1997;92:512-518.

25 Springer TA. Adhesion receptors of the immune system. Nature 1990;346:425-434.

26 Nelson PJ, Krensky AM. Chemokines, chemokine receptors, and allograft rejection. Immunity 2001;14: 377-386.

27 Bianchi E, Bender JR, Blasi F, et al. Through and beyond the wall: late steps in leukocyte transendothelial migration. Immunol Today 1997;18:586-591.

28 Vestweber D. Regulation of endothelial cell contacts during leukocyte extravasation. Curr Opin Cell Biol 2002;14:587-593.

29 Yoshimura T, Matsushima K, Tanaka S, et al. Purification of a human monocyte-derived neutrophil chemo- tactic factor that has peptide sequence similarity to other host defense cytokines. Proc Natl Acad Sci USA 1987;84:9233-9237.

30 Cinamon G, Shinder V, Alon R. Shear forces promote lymphocyte migration across vascular endothelium bearing apical chemokines. Nat Immunol 2001;2: 515-522.

31 Shaw SK, Bamba PS, Perkins BN, et al. Real-time imaging of vascular endothelial-cadherin during leukocyte transmigration across endothelium. J Immunol 2001;167:2323-2330.

32 Ostermann G, Weber KS, Zernecke A, et al. JAM-1 is a ligand of the beta(2) integrin LFA-1 involved in transendothelial migration of leukocytes. Nat Immunol 2002;3:151-158.

33 Edgell CJ, McDonald CC, Graham JB. Permanent cell line expressing human factor VIII-related antigen established by hybridization. Proc Natl Acad Sci USA 1983;80:3734-3737.

34 Ali S, Fritchley SJ, Chaffey BT, Kirby JA. Contribution of the putative heparan sulfate-binding motif BBXB of RANTES to transendothelial migration. Glycobiology 2002;12:535-543.

35 Paavola CD, Hemmerich S, Grunberger D, et al. Monomeric monocyte chemoattractant protein-1 (MCP-1) binds and activates the MCP-1 receptor CCR2B. J Biol Chem 1998;273:33157-33165.

36 Edgell CJ, Haizlip JE, Bagnell CR, et al. Endothelium specific Weibel-Palade bodies in a continuous human cell line, EA.hy926. In Vitro Cell Dev Biol 1990;26: 1167-1172.

37 Mutin M, Dignat-George F, Sampol J. Immunologic phenotype of cultured endothelial cells: quantitative analysis of cell surface molecules. Tissue Antigens 1997;50:449-458.

38 Douglas MS, Rix DA, Dark JH, Talbot D, Kirby JA. Examination of the mechanism by which heparin antagonizes activation of a model endothelium by interferon-gamma (IFN-gamma). Clin Exp Immunol 1997;107:578-584.

39 Douglas MS, Rix DA, Kirby JA. Antigen presentation by endothelium: heparin reduces the immunogenicity of interferon-gamma-treated endothelial cells. Transpl Immunol 1997;5:233-235.

40 Douglas MS, Rix DA, Larnkjaer A, et al. Blockade of the interaction between interferon-gamma and endothelial glycosaminoglycans: a novel strategy for immunosuppression? Transplant Proc 1997;29: 1086-1088.

41 Randolph GJ, Furie MB. A soluble gradient of endogenous monocyte chemoattractant protein-1 promotes the transendothelial migration of monocytes in vitro. J Immunol 1995;155:3610-3618.

42 Ali S, Palmer AC, Fritchley SJ, et al. Multimerization of monocyte chemoattractant protein-1 is not required for glycosaminoglycan-dependent transendothelial chemotaxis. Biochem J 2001;358:737-745.

43 David G, Bai XM, Van der Schueren B, et al. Developmental changes in heparan sulfate expression: in situ detection with mAbs. J Cell Biol 1992;119: 961-975.

44 Kure S, Yoshie O. A syngeneic monoclonal antibody to murine Meth-A sarcoma (HepSS-1) recognizes heparan sulfate glycosaminoglycan (HS-GAG): cell density and transformation dependent alteration in cell surface HS-GAG defined by HepSS-1. J Immunol 1996;137: 3900-3908. 
45 Hoogewerf AJ, Kuschert GS, Proudfoot AE, et al. Glycosaminoglycans mediate cell surface oligomerization of chemokines. Biochemistry 1997;36: 13570-13578.

46 Ali S, Hardy LA, Kirby JA. Transplant immunobiology: a crucial role for heparan sulfate glycosaminoglycans? Transplantation 2003;75:1773-1782.

47 Tanaka Y, Adams DH, Shaw S. Proteoglycans on endothelial cells present adhesion-inducing cytokines to leukocytes. Immunol Today 1993;14:111-115.

48 Proudfoot AE, Fritchley S, Borlat F, et al. The BBXB motif of RANTES is the principal site for heparin binding and controls receptor selectivity. J Biol Chem 2001;276:10620-10626.

49 Ali S, Palmer AC, Banerjee B, et al. Examination of the function of RANTES, MIP-1alpha, and MIP-1beta following interaction with heparin-like glycosaminoglycans. J Biol Chem 2000;275:11721-11727.

50 Middleton J, Neil S, Wintle J, et al. Transcytosis and surface presentation of IL-8 by venular endothelial cells. Cell 1997;91:385-395.

51 Engling A, Backhaus R, Stegmayer C, et al. Biosynthetic FGF-2 is targeted to non-lipid raft microdomains following translocation to the extracellular surface of CHO cells. J Cell Sci 2002;115:3619-3631.

52 Carter NM, Ali S, Kirby JA. Endothelial inflammation: the role of differential expression of $N$-deacetylase $/ N$ sulphotransferase enzymes in alteration of the immunological properties of heparan sulfate. J Cell Sci 2003;116:3591-3600.

53 Gotte M. Syndecans in inflammation. FASEB J 2003;17:575-591. 